\title{
Acrodermatitis enteropática: caso clínico y revisión de la literatura
}

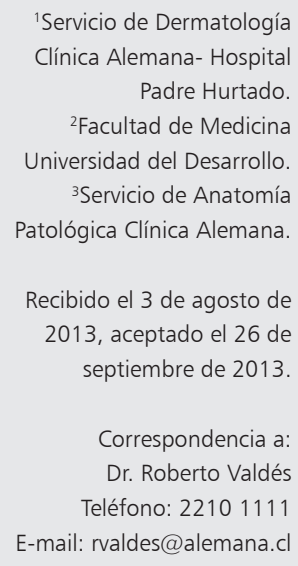

\author{
ROBERTO VALDÉS ${ }^{1}$, MICHELLE MAURET ${ }^{2}$, ÁLEX CASTRO ${ }^{3}$
}

Acrodermatitis enteropathica is an uncommon disease caused by hereditary or acquired zinc deficiency. It is characterized by a triad of alopecia, diarrhea and acral and periorificial dermatitis. It is treated with Zinc supplementation. We report a 31-year-old indigent and drug addict female with a cutaneous-mucous syndrome characteristic of acrodermatitis enteropathica. She had a positive clinical evolution after Zinc supplementation.

(Rev Med Chile 2013; 141: 1480-1483)

Key words: Acrodermatitis; Zinc deficiency.
$\mathrm{P}$ aciente de sexo femenino, 31 años, indigente, con antecedentes de poliadicción a sustancias de abuso y alcohol pero sin antecedentes médicos conocidos.

Consultó por lesiones peribucales dolorosas de un mes de evolución que rápidamente se extendieron al resto de la cara. $\mathrm{Al}$ inicio del cuadro presentó diarrea autolimitada y una semana previa a los síntomas mantuvo ingesta excesiva de alcohol y drogas.

Al ingreso presenta placas eritematovioláceas, hiperqueratósicas, con descamación gruesa en cara, cuello, genitales y segmentos distales de las extremidades en forma simétrica (Figuras 1 y 2 ).

Se plantea una toxicodermia fija intensa y acrodermatitis enteropática. Se solicita exámenes donde destaca leucocitos $10.600 \mathrm{x} \mathrm{mm}^{3}, \mathrm{Hb} 13 \mathrm{~g} /$ dl, Creatinina 0,39 mg/dl, BUN $7 \mathrm{mg} / \mathrm{dl}$, Proteínas totales 5,6 g/dl, Albúmina 2,7 g/dl, RPR no reactivo, VIH (-) VHB (-) VHC (-). Zinc plasmático $25 \mathrm{ug} / \mathrm{dL}(\mathrm{VN}>50)$. En la biopsia se observó hiperortoqueratosis con focos de paraqueratosis, hipogranulosis, hiperplasia epidérmica psoriasiforme y focos de necrosis confluente con algunas vesículas intraepidérmicas, dilatación vascular y leve infiltrado linfocitario perivascular en la dermis papilar (Figura 3).
Se confirmó diagnóstico de acrodermatitis enteropática. Se inició régimen adecuado con suplementación de zinc con lo cual el cuadro revirtió de manera progresiva durante la hospitalización.

\section{Acrodermatitis enteropática}

Trastorno bioquímico y nutricional, poco frecuente, producido por la deficiencia de zinc ${ }^{1}$. Fue reconocida por primera vez en 1936 por Brandt, dermatólogo sueco. En 1974, Moynahan destacó por primera vez el papel de la deficiencia de zinc y la ausencia de una oligopeptidasa del intestino como responsable de la disminución sérica.

La deficiencia de zinc puede ser heredada o adquirida. Se caracteriza clínicamente por la presencia de lesiones en zonas distales de la piel o periorificial, alopecia y diarrea ${ }^{1,2,5}$.

La forma heredada de la acrodermatitis enteropática $(\mathrm{AE})$ corresponde a un defecto genético autosómico recesivo, causado por la mutación del gen SLC39A4 localizado en el cromosoma 8q24.3; este gen que codifica las proteínas trasportadoras de zinc, entre ellas ZIP4, proteína que permite la adecuada absorción entérica de zinc. Se han reportado 19 mutaciones que pueden causar acrodermatitis enteropática ${ }^{5,6}$. 
Típicamente, se presenta en lactantes y coincide con la suspensión de la lactancia materna. Se cree que la leche humana contiene ligandos que benefician la absorción del zinc, lo cual enmascara el cuadro durante la lactancia ${ }^{6}$.

En la forma adquirida la deficiencia de zinc es producida por falta de ingesta, aumento de la excreción o mala absorción en la dieta ${ }^{9}$.

Otras circunstancias asociadas a la deficiencia de zinc incluyen carencia en la dieta, dieta rica en fitatos y calcio, vegetarianismo, anorexia, ausencia de depósitos de zinc en prematuros, cirrosis hepática, alcoholismo donde su consumo crónico disminuye la absorción intestinal de zinc y aumenta su excreción urinaria, por otro lado, se agrega la baja ingesta de alimentos en estos pacientes; síndromes de malabsorción intestinal, cirugía gástrica, enteropatía inducida por fármacos, insuficiencia pancreática, anemia perniciosa, grandes quemados, síndrome nefrótico, VIH y fibrosis quística ${ }^{9,11}$.

La AE heredada tiene una incidencia estimada de 1 por cada 500.000 niños, sin predilección por raza o sexo. La deficiencia adquirida por falta de ingesta afecta un tercio de la población en el sureste de Asia y África subsahariana, donde los niños son los más afectados. En América Latina y el Caribe se registran 53 millones de personas desnutridas que están expuestas a padecer esta enfermedad. La suplementación de zinc ha demostrado reducir la morbi-mortalidad infantil ${ }^{8}$.

\section{Metabolismo y funciones del zinc}

El zinc se encuentra principalmente en alimentos de origen animal. También está presente en la leche materna. La absorción se produce en duodeno y yeyuno. Su excreción es principalmente intestinal ${ }^{7,12}$.

El zinc juega un papel importante en el metabolismo de proteínas, carbohidratos y vitaminas en el crecimiento, desarrollo y proliferación celular, así como en la curación y reparación de los tejidos. Además es cofactor enzimático ${ }^{7,12}$.

Se ha demostrado que la concentración de zinc influye en diversos mecanismos inmunológicos mediados por células, importantes en la prevención de infecciones mucocutáneas ${ }^{10,12}$.

Algunos autores plantean que el zinc es necesario para la producción de ceruloplasmina, por lo tanto, lleva a una pobre biodisponibilidad de cobre, alterando su función normal como fungicida $^{7,10}$.

\section{Clínica}

Los hallazgos cutáneos clásicos incluyen una erupción simétrica, eritematosa, descamativa, eccematosa, vesículo-ampollosa o purulenta sobre las superficies distales y periorificiales ${ }^{1}$ que, en algunos casos, pueden simular una dermatitis atópica o una psoriasis. Además pueden presentar cambios en el color del pelo, paroniquia y hasta pérdida ungueal. Sin tratamiento, las lesiones de la piel lentamente evolucionan a erosiones y los pacientes desarrollan alopecia generalizada y diarrea. La tríada clásica, que sólo se presenta en $20 \%$ de los pacientes, incluye dermatitis con localización periorificial y distal, alopecia total y diarrea ${ }^{1,3,9}$.

En la enfermedad avanzada, los pacientes pueden experimentar: retardo en la cicatrización de heridas, anemia, disgeusia, hiposmia, retardo de la pubertad, hipogonadismo en niños y hombres, retraso en el crecimiento y retardo mental, trastornos psicológicos con cambios de humor, anorexia $\mathrm{y}$ alteraciones del comportamiento ${ }^{1,3}$.

Son sensibles a la sobreinfección por gérmenes como cándida y bacterias como el estafilococo aureus, que suele ser una grave complicación de esta patología ${ }^{7,10}$.

\section{Diagnóstico}

El diagnóstico de la AE es clínico y debe considerar la historia nutricional. Se confirma con la demostración de una baja concentración de zinc plasmático o en los eritrocitos, siendo el nivel sérico el más utilizado para el diagnóstico; su valor normal está entre 70 y $150 \mu \mathrm{g} / \mathrm{dl}$ y en los eritrocitos entre 440 y $860 \mu \mathrm{g} / \mathrm{dl}^{1,3}$.

En la evaluación histológica de la piel, los hallazgos característicos incluyen hiperplasia psoriasiforme con paraqueratosis confluente, espongiosis, palidez de la epidermis superior, apoptosis y atrofia epidérmica variable con disminución o ausencia del estrato granuloso. Las lesiones vesículo-ampollares son el resultado de degeneración vacuolar de los queratinocitos epidérmicos, con balonamiento, citolisis y degeneración reticular o producto de necrosis confluente. En ocasiones se 


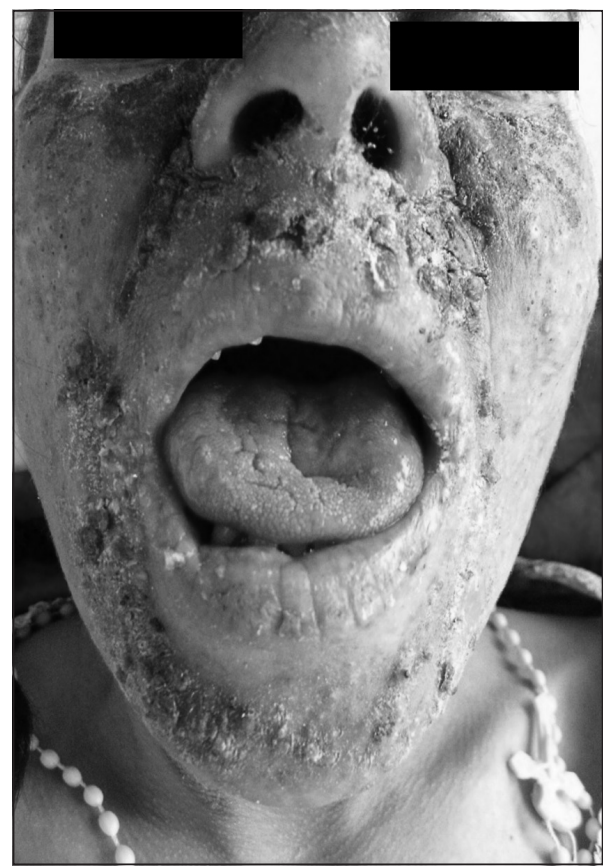

Figura 1. Lesiones peribucales dolorosas e hiperqueratósicas en zona facial.

observan pústulas subcórneas, que por lo general indican infección secundaria. En la dermis papilar se observa dilatación vascular e infiltrado linfocitario perivascular. En la etapa inicial los cambios son sutiles y pueden limitarse a focos de paraqueratosis que alternan con ortoqueratosis. Estos hallazgos no son patognomónicos ${ }^{1,9}$.

\section{Diagnóstico diferencial}

El diagnóstico diferencial incluye psoriasis, dermatitis atópica, dermatitis del pañal, candidiasis, dermatitis seborreica, fibrosis quística, histiocitosis de células de Langerhans, glucagonoma, pelagra e hipovitaminosis ${ }^{1}$.

También debe considerarse deficiencia de biotina, isoleucina, acidemia metilmalónica y propiónica, fenilcetonuria, enfermedad de Hartnup, enfermedad de Leiner, síndrome de Wiskott-Aldrich, inmunodeficiencia combinada grave, queratodermia palmo-plantar y periorificial congénito ${ }^{1,3}$.

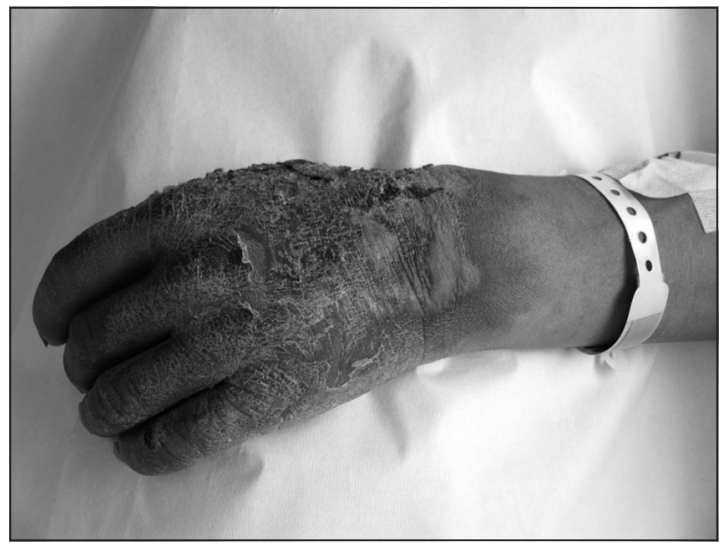

Figura 2. Placas eritematovioláceas, hiperqueratósicas, con descamación gruesa en zona acral, simétricas.

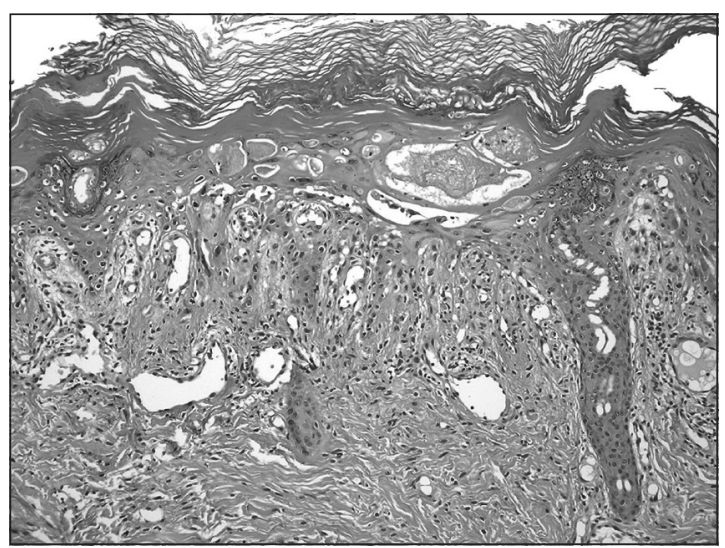

Figura 3. Microfotografía: Se observa marcada hiperortoqueratosis con focos de paraqueratosis, hipogranulosis, hiperplasia epidérmica psoriasiforme y focos de necrosis confluente con algunas vesículas intraepidérmicas, dilatación vascular y leve infiltrado linfocitario perivascular en la dermis papilar HE 200x.

\section{Tratamiento}

El tratamiento oral con zinc es el de elección. El zinc elemental puede administrarse como sulfato para mejor tolerancia. Estos suplementos llevan a una rápida mejoría clínica en días o semanas, con la resolución de las lesiones cutáneas y, en un plazo de 2 a 4 semanas, con el comienzo de recrecimiento del pelo ${ }^{1,9,11}$.

En la $\mathrm{AE}$ adquirida, se debe iniciar con 0,5 a 1 
$\mathrm{mg} / \mathrm{kg}$ al día para niños y 15 a $30 \mathrm{mg}$ al día para los adultos; además, se recomienda orientación nutricional que incluya el consumo de proteínas animales, considerando que sustancias como los fitatos, que están presentes en cereales y vegetales, son poderosos quelantes del zinc y modifican su absorción. Otras sustancias que bloquean la absorción son la tetraciclina, la penicilina, los esteroides, el hierro y el calcio en altas dosis, el alcohol, el tanino y los anticonceptivos orales. La ausencia de tratamiento hace de la AE una enfermedad mortal ${ }^{1,11}$.

Los efectos adversos de la ingestión de zinc, incluyen náuseas, vómito, hemorragia gastrointestinal; letargo, neuropatía, deshidratación. La sobredosis accidental de zinc puede causar una falla multisistémica ${ }^{11}$.

Como conclusión, se debe destacar la importancia de pensar en el diagnóstico de AE en todo paciente que se presente con diarrea, irritabilidad y lesiones periorificiales o en zonas acrales, pues si instalamos un tratamiento precoz, evitaremos complicaciones.

\section{Referencias}

1. Wang LC, Busbey S. Images in clinical medicine. Acquired acrodermatitis enteropathica. N Engl J Med 2005; 352 (11): 1121.

2. Chen MD, Song YM. An infant with acrodermatitis enteropathica-like symptoms but without hypozincemia. Pediatr Dermatol 2005; 22 (3): 280-1.
3. Perafan-Riveros C, Franca LF, Alves AC, Sanches JA Jr. Acrodermatitis enterophatica: case report and review of the literature. Peditr Dermatol 2002; 19 (5): 426-31.

4. Radja N, Charles-Holmes R. Acrodermatitis enteropathica lifelong follow-up and zinc monitoring. Clin Exp Dermatol 2002; 27 (1): 62-3.

5. Wang K, Zhou B, Kuo YM, Zemansky J, Gitschier J. A novel member of a zinc transporter family is defective in acrodermatitis enteropathica. Am J Hum Genet 2000; 71: 66-73.

6. Vardi A, Anikster Y, Eisenkraft A, Shohat M, Abu-Much J, Eisenkraft S, et al. A new genetic isolate of acrodermatitis enteropathica with a novel mutation. Br J Dermatol 2009; 160: 1346-8.

7. Maverakis E, Fung MA, Lynch PJ. Acrodermatitis enteropathica and an overview of zinc metabolism. J Am Acad Dermatol 2007; 56: 116-24.

8. Brandt T. Dermatitis in children with disturbances of general condition and absence of food. Acta Derm Venereol 1936; 17: 513-6.

9. Richlin VA, García-Zuazaga JA, Beck MJ. What is your diagnosis? Acquired acrodermatitis enteropathica. Cutis 2008; 81: 314, 324-6.

10. Fischer Walker C, Black RE. Zinc and the risk for infectious disease. Annu Rev Nutr 2004; 24: 255-75.

11. Bales CW, DiSilvestro RA, Currie KL, Plaisted CS, Joung $\mathrm{H}$, Galanos AN, et al. Marginal zinc deficiency in older adults: responsiveness of zinc status indicators. J Am Coll Nutr 1994; 13: 455-62.

12. López de Romaña D, Castillo C, (2), Diazgranados D, El Zinc en la salud humana II, Rev Chil Nutr 2010; 37 (2) Santiago. 